\title{
Attribute Exploration with Background Implications and Exceptions
}

\author{
Gerd Stumme \\ Technische Hochschule Darmstadt, Fachbereich Mathematik \\ Schloßgartenstr.7, D-64289 Darmstadt, stumme@mathematik.th-darmstadt.de \\ (C) Springer-Verlag Berlin-Heidelberg 1995 \\ Summary: Implications between attributes can represent knowledge about ob- \\ jects in a specified context. This knowledge representation is especially useful \\ when it is not possible to list all specified objects. Attribute exploration is a tool \\ of formal concept analysis that supports the acquisition of this knowledge. For a \\ specified context this interactive procedure determines a minimal list of valid im- \\ plications between attributes of this context together with a list of objects which \\ are counterexamples for all implications not valid in the context. This paper de- \\ scribes how the exploration can be modified such that it determines a minimal \\ set of implications that fills the gap between previously given implications (called \\ background implications) and all valid implications. The list of implications can \\ be simplified further if exceptions are allowed for the implications.
}

\section{Introduction}

Implications between attributes can represent knowledge about objects in a specified context. This knowledge representation is especially useful when it is not possible to list all specified objects. Attribute exploration (cf. Ganter (1987), Wille (1989)) is a tool of formal concept analysis that supports the acquisition of this knowledge. Formal concept analysis was introduced in Wille (1982) and has grown during the last fifteen years to an useful tool in data analysis.

For a specified context the interactive procedure of attribute exploration determines a minimal list of implications that is sufficient to deduce all valid implications between the attributes of the context together with a list of objects which are counterexamples for the implications that are not valid in the context. As an interactive procedure, the program suggests implications to the user which do not contradict to already given counterexamples. The user can either accept a suggested implication or he must supply a new object as a counterexample.

Usually, the user does not start such an exploration from scratch, but he has already some idea how the attributes are related. Before starting the exploration he can enter a list of objects which may significantly decrease the number of remaining possible implications.

Another possibility is to enter some already known implications before starting the exploration. These implications, the user already knows to be valid, 
are here called background implications. This paper describes a generalization of attribute exploration, that it is able to determine a minimal list of implications which - together with the background implications - is sufficient to deduce all valid implications.

In P. Burmeisters implementation (1987), it is also possible to enter background implications. This program however determines a minimal set of implications - regardless of the background implications. They are only used to decrease the number of questions to the user. The resulting list in this program does not depend in any way on the background implications one starts with. In the approach presented in this paper the background implications are used to minimize only the number of additionally needed implications. This is based on the belief that it is not always the best to describe a context by a minimal number of implications. There may be more implications if some of them are obvious.

In the next section the basic definitions of formal concept analysis are recalled and explained by an example, before implications in contexts and the notions of completeness and irredundancy are introduced. In the third section the interactive procedure of attribute exploration with background implications is described. It is illuminated by an example in the last section, where we also discuss, how the admission of exceptions can further simplify the result of the exploration.

\section{Implications of Contexts and the $\mathcal{L}$-Duquenne- Guigues-basis}

First we briefly recall the basic definitions of formal concept analysis (cf. Ganter and Wille (1995)) and give an example.

Definition: We call $(G, M, I)$ a context, where $G$ and $M$ are sets and $I$ is a relation between $G$ and $M$ (i. e. $I \subseteq G \times M)$. The elements of $G$ and $M$ are called objects and attributes, respectively, and $g \operatorname{Im}(: \Longleftrightarrow(g, m) \in I)$ is read: "the object $g$ has the attribute $m$ ".

For every set $A \subseteq G$ of objects we define the set $A^{\prime}:=\{m \in M \mid g I m$ for all $g \in A\}$ of all attributes shared by all objects in $A$. Dually the set $B^{\prime}:=\{g \in G \mid g I m$ for all $m \in B\}$ is the set of all objects having all attributes in $B \subseteq M$.

Now a concept of the context $(G, M, I)$ is a pair $(A, B)$ with $A \subseteq G, B \subseteq M$, $A^{\prime}=B$, and $B^{\prime}=A$. The set $A$ is called the extent of the concept, the set $B$ the intent. The hierarchical subconcept-superconcept-relation is given by $\left(A_{1}, B_{1}\right) \leq\left(A_{2}, B_{2}\right): \Longleftrightarrow A_{1} \subseteq A_{2}\left(\Longleftrightarrow B_{1} \supseteq B_{2}\right)$. The set of all concepts of a context $(G, M, I)$ together with this order relation is a complete lattice which is called the concept lattice of $(G, M, I)$ and is denoted by $\underline{\mathfrak{B}}(G, M, I)$.

Infimum and supremum in the concept lattice are calculated as follows:

$$
\bigwedge_{t \in T}\left(A_{t}, B_{t}\right)=\left(\bigcap_{t \in T} A_{t},\left(\bigcup_{t \in T} B_{t}\right)^{\prime \prime}\right), \quad \bigvee_{t \in T}\left(A_{t}, B_{t}\right)=\left(\left(\bigcup_{t \in T} A_{t}\right)^{\prime \prime}, \bigcap_{t \in T} B_{t}\right) .
$$


The following example shows how the concept lattice unfolds the conceptual relationships contained in the underlying data context: We consider the formal context shown in Fig. 1. Its objects are the graphs $G_{1}$ up to $G_{18}$ and its attributes are ten attributes of undirected graphs (cf. Wilson (1975)): connected, disconnected, bipartite, complete, complete bipartite, tree, forest, planar, Eulerian, Hamiltonian. This context (together with a list of implications) is the result of the attribute exploration that is described in the third section.

It is sufficient to label the line diagram not with the complete concepts, but only with the attributes and objects: For every object $g$, its object concept $\gamma(g)$, which is defined as the concept with the smallest extent containing $g$, is labeled with " $g$ ". Dually, for every attribute $m$ its attribute concept $\mu(m)$, which is defined as the concept with the smallest intent containing $m$, is labeled with " $m$ ". Then the extent and intent of every concept can be determined in the diagram: A concept contains all those objects in its extent which are linked to it by a descending path and it contains all those attributes in its intent that are linked to it by an ascending path. The rightmost concept in Fig. 2 for example has the graphs $G_{3}, G_{14}$ and $G_{18}$ in its extent and the attributes connected, complete, Eulerian, and Hamiltonian in its intent.

In the diagram one can see two cubes at the top: one is spanned by $\mu$ (disconnected $), \mu($ planar $)$ and $\mu$ (bipartite), and the other by $\mu$ (connected $), \mu($ planar $)$ and $\mu$ (bipartite). This indicates that in both cases the three involved attributes are independent.

The dominating part in the lattice lies between $\mu$ (connected) and $\gamma\left(G_{13}\right)$. It is the direct product of a 6-element "ladder" with a 4-element "rectangle", but it can also be seen as 4-dimensional hypercubes that are glued together at eight vertices. The upper one lies between $\mu$ (connected) and $\gamma\left(G_{15}\right)$ and is spanned by $\mu($ planar $) \wedge \mu($ connected $), \mu($ bipartite $) \wedge \mu($ connected $), \mu($ Eulerian), and $\mu$ (Hamiltonian). This shows that, for connected graphs, the four attributes planar, bipartite, Eulerian, and Hamiltonian are independent.

In the lower hypercube (between $\mu$ (bipartite) $\wedge \mu($ connected $)$ ) and $\gamma\left(G_{13}\right)$ one point is missing - it is marked in the diagram with a little dot left of $\gamma\left(G_{15}\right)$. This indicates that every complete bipartite Hamiltonian planar graph is also Eulerian. In fact there exist (up to isomorphism) only two such graphs, $G_{13}$ and $G_{18}$.

Definition: An implication between attributes in $M$ is a pair $(X, Y)$ of subsets $X$ and $Y$ of $M$. It is denoted by $X \rightarrow Y$ and is read " $X$ implies $Y$ ". A subset $T$ of $M$ respects the implication if $X \nsubseteq T$ or $Y \subseteq T$. The set $T$ respects a set $\mathcal{L}$ of implications if it respects every implication in $\mathcal{L}$. An implication $X \rightarrow Y$ is valid in a context $\mathbb{K}$ if it is respected by every object intent. The implication is then called an implication of the context $\mathbb{K}$. An implication $X \rightarrow Y$ is entailed by a set $\mathcal{L}$ of implications if every subset of $M$ that respects $\mathcal{L}$ also respects $X \rightarrow Y$. 

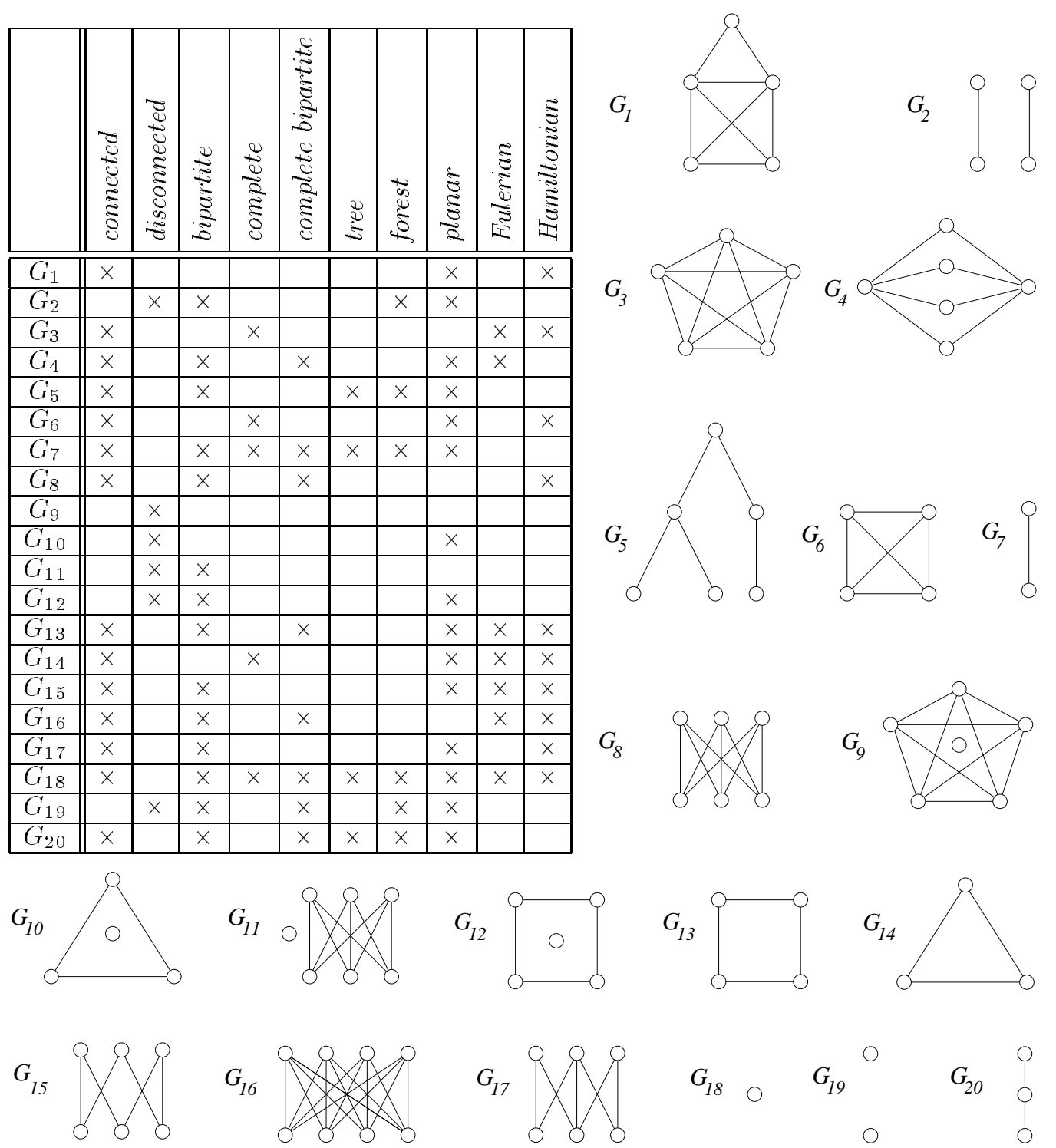

$$
\begin{array}{llllll}
G_{18} & \circ & G_{19} & & & \\
& & & &
\end{array}
$$

Figure 1: Context of graphs

Lemma 1 An implication $X \rightarrow Y$ is valid in a context $\mathbb{K}$ if and only if $Y \subseteq X^{\prime \prime}$. Then it is also respected by every concept intent of $\mathbb{K}$.

The structure of a concept lattice is already described by all implications between the attributes of the context, because the set of all intents is exactly the largest closure system on $M$ that respects all these implications. The implications can equivalently be understood as equalities in the $\Lambda$-semilattice $\underline{\mathfrak{B}}(\mathbb{K}):$ In our example $\{$ complete, Eulerian $\} \rightarrow\{$ Hamiltonian $\}$ is an implication of the context, which corresponds to the equality $\mu$ (complete $) \wedge$ $\mu($ Eulerian $)=\mu($ complete $) \wedge \mu($ Eulerian $) \wedge \mu($ Hamiltonian $)$ in the concept lattice. 


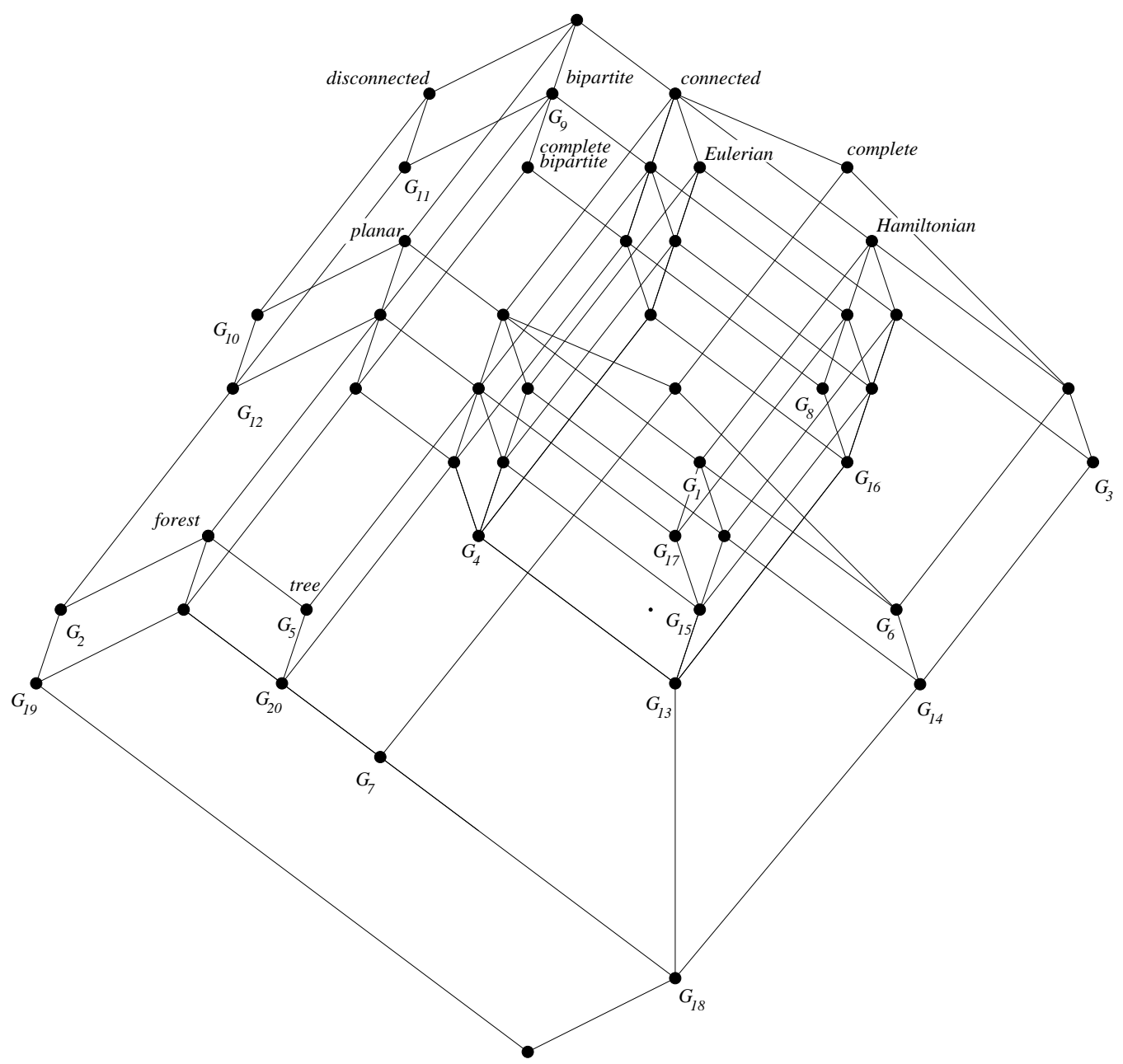

Figure 2: Concept lattice of the context in Fig. 1

Some of these implications may be known in advance, and in this paper they will be referred to as background implications. In a formal sense every implication of a context can be a background implication. The question is now how to describe the structure of the concept lattice with implications in the most efficient way when background implications are given. We are looking for a minimal list that is "filling the gap" between the background implications and all valid implications.

Definition: Let $\mathbb{K}$ be a finite context and $\mathcal{L}$ a set of (background) implications of $\mathbb{K}$. A set $\mathcal{B}$ of implications of $\mathbb{K}$ is called $\mathcal{L}$-complete, if every implication of $\mathbb{K}$ is entailed by $\mathcal{L} \cup \mathcal{B}$. It is called $\mathcal{L}$-irredundant if no implication $A \rightarrow B \in \mathcal{B}$ is entailed by $(\mathcal{B} \backslash\{A \rightarrow B\}) \cup \mathcal{L}$. A $\mathcal{L}$-basis is a $\mathcal{L}$-complete and $\mathcal{L}$-irredundant set of implications of $\mathbb{K}$. If $\mathcal{L}$ is empty then $\mathcal{B}$ is called complete, irredundant, and a basis, respectively. A subset $P$ of $M$ is called pseudo-intent of $\mathbb{K}$ if $P \neq P^{\prime \prime}$ and if for every pseudo-intent $Q$ with $Q \subset P$ the inclusion $Q^{\prime \prime} \subseteq P$ holds. 
J.-L. Guigues and V. Duquenne (1986) show that $\mathcal{B}:=\left\{P \rightarrow P^{\prime \prime} \mid P\right.$ is a pseudointent $\}$ is a basis of $(G, M, I)$ if $M$ is finite. This basis is called Duquenne-Guigues-basis. We obtain a $\mathcal{L}$-basis by generalizing this definition.

Without further mentioning, all sets of attributes considered in the following will be finite.

Definition: The closure operator on the set $M$ of attributes induced by the background implications is denoted by $P \mapsto \bar{P}:=P \cup P^{\mathcal{L}} \cup P^{\mathcal{L L}} \cup \ldots$ with $X^{\mathcal{L}}:=X \cup \cup\{B \subseteq M \mid A \subseteq X, A \rightarrow B \in \mathcal{L}\}$.

A subset $P$ of $M$ is called $\mathcal{L}$-pseudo-intent of $\mathbb{K}$ if $P=\bar{P} \neq P^{\prime \prime}$ and if, for every $\mathcal{L}$-pseudo-intent $Q$ with $Q \subset P$, the inclusion $Q^{\prime \prime} \subseteq P$ holds. The set $\mathcal{B}_{\mathcal{L}}:=\left\{P \rightarrow P^{\prime \prime} \mid P\right.$ is a $\mathcal{L}$-pseudo-intent $\}$ of implications is called $\mathcal{L}$-Duquenne-Guigues-basis.

Theorem $2 \mathcal{B}_{\mathcal{L}}$ is a $\mathcal{L}$-basis of $\mathbb{K}$.

Proof. Obviously, all implications in $\mathcal{B}_{\mathcal{L}}$ are implications of $\mathbb{K}$. We prove that $\mathcal{B}_{\mathcal{L}}$ is $\mathcal{L}$-complete by showing that every subset $T \subseteq M$ respecting all implications in $\mathcal{L} \cup \mathcal{B}_{\mathcal{L}}$ is an intent: As $T \rightarrow \bar{T}$ is entailed by $\mathcal{L}$ we have $T=\bar{T}$. Furthermore $T$ respects $Q \rightarrow Q^{\prime \prime}$ for every $\mathcal{L}$-pseudo-intent $Q \subseteq T$. Suppose $T \neq T^{\prime \prime}$. Then $T$ is a $\mathcal{L}$-pseudo-intent by definition and so $T \rightarrow T^{\prime \prime} \in \mathcal{B}_{\mathcal{L}}$. This is a contradiction because $T$ does not respect this implication.

Let $P \rightarrow P^{\prime \prime} \in \mathcal{B}_{\mathcal{L}}$. We show that $P \rightarrow P^{\prime \prime}$ is not entailed by $\left(\mathcal{B}_{\mathcal{L}} \backslash\right.$ $\left.\left\{P \rightarrow P^{\prime \prime}\right\}\right) \cup \mathcal{L}$ because $P$ respects all implications in $\left(\mathcal{B}_{\mathcal{L}} \backslash\left\{P \rightarrow P^{\prime \prime}\right\}\right) \cup \mathcal{L}$ (and prove so that $\mathcal{B}_{\mathcal{L}}$ is $\mathcal{L}$-irredundant): As $P=\bar{P}$, it clearly respects all implications in $\mathcal{L}$. For $Q \rightarrow Q^{\prime \prime} \in \mathcal{B}_{\mathcal{L}} \backslash\left\{P \rightarrow P^{\prime \prime}\right\}$ with $Q \subseteq P$ we have $Q^{\prime \prime} \subseteq P$, as $P$ is a $\mathcal{L}$-pseudo-intent. Hence $P$ respects also $Q \rightarrow Q^{\prime \prime}$.

One may ask if it is possible to get the $\mathcal{L}$-pseudo-intents by just closing the pseudo-intents with ${ }^{-}$and then deleting the (trivial) implications of the form $A \rightarrow A$. The context in Fig. 3 shows that in general this is not the case. The Duquenne-Guigues-basis is $\mathcal{B}=\{c d \rightarrow a b c d, b \rightarrow a b, a d \rightarrow a b c d, a c \rightarrow a b c d\}$. For the background implication $\mathcal{L}:=\{c d \rightarrow a\}$ we get the $\mathcal{L}$-Duquenne-Guiguesbasis $\mathcal{B}_{\mathcal{L}}=\{b \rightarrow a b, a d \rightarrow a b c d, a c \rightarrow a b c d\}$ while $\left\{\bar{P} \rightarrow P^{\prime \prime} \mid P\right.$ is pseudo-intent with $\left.\bar{P} \neq P^{\prime \prime}\right\}$ additionally contains $a c d \rightarrow a b c d$. In general the resulting set is not $\mathcal{L}$-irredundant.

\section{Attribute Exploration with Background Implications}

B. Ganter (1987) presents attribute exploration as an interactive knowledge acquisition tool that can be used to determine the Duquenne-Guigues-basis of a context that is either too large for a complete input into the computer or that is even not completely known. It is based on his Next-ClosureAlgorithm that efficiently calculates closure systems. 


\begin{tabular}{|c||c|c|c|c|}
\hline & $a$ & $b$ & $c$ & $d$ \\
\hline \hline 1 & $\times$ & & & \\
\hline 2 & $\times$ & $\times$ & & \\
\hline 3 & & & $\times$ & \\
\hline 4 & & & & $\times$ \\
\hline
\end{tabular}

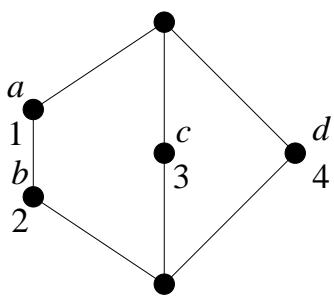

Figure 3:

The Attribute Exploration procedure can be modified such that it can be used to determine interactively the $\mathcal{L}$-Duquenne-Guigues-basis for a given set $\mathcal{L}$ of background implications. Therefore we proceed similar to Ganter (1987). First we show that the set of all intents and $\mathcal{L}$-pseudo-intents is a closure system on the set $M$ of all attributes:

Lemma 3 Let $(G, M, I)$ be a context, let $\mathcal{L}$ be a set of implications of $(G, M, I)$, and let $P$ and $Q$ be intents or $\mathcal{L}$-pseudo-intents with $P \nsubseteq Q$ and $Q \nsubseteq P$. Then $P \cap Q$ is an intent.

Proof. $P$ as $Q$ and therefore also $P \cap Q$ respect all implications in $\mathcal{L} \cup \mathcal{B}_{\mathcal{L}}$ except $P \rightarrow P^{\prime \prime}$ and $Q \rightarrow Q^{\prime \prime}$. Because of $P \nsubseteq P \cap Q$ and $Q \nsubseteq P \cap Q$ the set $P \cap Q$ respects these implications, too. Hence it must be an intent.

Corollary 4 The set of all intents and $\mathcal{L}$-pseudo-intents of a finite context $(G, M, I)$ is a closure system on $M$; with the closure operator $X \mapsto X^{\bullet}:=$ $X \cup X^{\circ} \cup X^{\circ \circ} \cup \ldots$, where $X^{\circ}:=\overline{X \cup \cup\left\{B \subseteq M \mid A \rightarrow B \in \mathcal{B}_{\mathcal{L}}, A \subset X\right\}}$.

Next we introduce the lectical order on the set of subsets of $M$. For the sake of simplicity we assume that $M:=\{1, \ldots, n\}$.

Definition: The lectical order on $\mathfrak{P}(M)$ is defined by $A<B: \Longleftrightarrow(\exists i \in B \backslash A: A \cap\{1, \ldots, i-1\}=B \cap\{1, \ldots, i-1\})$ for $A, B \subseteq M$.

For $A, B \subseteq M$ and $i \in M$ we define

$A<_{i} B: \Longleftrightarrow(i \in B \backslash A$ and $A \cap\{1, \ldots, i-1\}=B \cap\{1, \ldots, i-1\})$ and $A \oplus i:=((A \cap\{1, \ldots, i-1\}) \cap\{i\})^{\bullet}$.

The Next-Closure-algorithm of B. Ganter (1987) lists all closed sets of a closure system on a finite set in the lectical order. In the next theorem it is applied to the closure system of all intents and all $\mathcal{L}$-pseudo-intents:

Theorem 5 The lectically first intent or $\mathcal{L}$-pseudo-intent is $\bar{\emptyset}$. For a given subset $B \subseteq M$ the lectically next intent or $\mathcal{L}$-pseudo-intent is the set $B \oplus i$, where $i$ is the maximal element in $M \backslash B$ with $B<_{i} B \oplus i$. The lectically last intent or $\mathcal{L}$-pseudo-intent is $M$. 
This theorem provides the central part of the Attribute Exploration with background implications, which we describe now: We want to determine the $\mathcal{L}$-Duquenne-Guigues-basis of a context $(G, M, I)$ (which is a priori not completely given) for a set $\mathcal{L}$ of background implications of the context. The algorithm starts with a partial context $(H, M, I \cap(H \times M)$ for a subset $H \subseteq G$ of objects. The set $H$ may also be empty.

Algorithm. Set $k:=1$ and $\mathcal{B}_{\mathcal{L}}:=\emptyset$.

(1) Determine the $k$ th $\mathcal{L}$-pseudo-intent $P_{k}$ of $(H, M, I \cap(H \times M)$ by applying Theorem 5. If $M$ is reached (as intent) then STOP. $\mathcal{B}_{\mathcal{L}}$ is then the $\mathcal{L}$-Duquenne-Guigues-basis.

(2) Ask the user: "Is the implication $P_{k} \rightarrow P_{k}^{\prime \prime}$ valid?"

- If the answer is "Yes", then add $P_{k} \rightarrow P_{k}^{\prime \prime}$ to $\mathcal{B}_{\mathcal{L}}$ and increase $k$.

- If the answer is "No", then ask for an object $g$ that does not respect this implication. The row $\{g\}^{\prime}$ then also has to be entered by the user. Add $g$ to $H$.

(3) Go to step (1).

The algorithm is correct because the lectically first $\mathcal{L}$-pseudo-intents do not change when an object is added that respects all previously accepted implications and all background implications:

Theorem 6 Let $(H, M, J)$ be a finite context and let $P_{1}, \ldots, P_{k}$ be the $k$ lectically first $\mathcal{L}$-pseudo-intents of $(H, M, J)$. Let $(G, M, I)$ be a finite context with $H \subseteq G$ and $J=I \cap(H \times M)$, in which all the implications in $\mathcal{L} \cup\left\{P_{i} \rightarrow\right.$ $\left.P_{i}^{J J} \mid i=1, \ldots, k\right\}$ are valid. Then $P_{1}, \ldots, P_{k}$ are also the $k$ lectically first $\mathcal{L}$-pseudo-intents of $(G, M, I)$.

Proof. For $i=1, \ldots, k$ we have $P_{i}^{I I}=P_{i}^{J J}$ because for every $g \in G$ the set $\{g\}^{I}$ respects all $P_{i} \rightarrow P_{i}^{J J}$. As every $\mathcal{L}$-pseudo-intent $Q \subset P_{j}$ of $(H, M, J)$ is lectically less than $P_{j}$, the assertion is a consequence of the definition of $\mathcal{L}$-pseudo-intent.

\section{An Exploration of Graphs}

In this section we see how the context in Fig. 1 is produced. We start with the set $M:=\{$ connected (conn), disconnected (disc), bipartite (bip), complete (comp), complete bipartite (cbip), tree (tree), forest (for), planar (plan), Eulerian (eul), Hamiltonian (ham)\} of attributes and want to know which implications between these attributes are valid for all undirected graphs. As the class $G$ of undirected graphs contains infinitely many isomorphism classes, there is no possibility to determine the Duquenne-Guigues-basis for 
the infinite context $(G, M, I)$ directly. One has to work with some "typical" graphs. The graphs the user gives as counterexamples during the attribute exploration are just these typical graphs.

When we look at the list of attributes then we see that connected and disconnected are contradicting each other (i. e., no graph can have both attributes). Furthermore we know that a tree is just defined as a connected forest and that a complete bipartite graph is always bipartite. This justifies the following background implications:

$$
\begin{aligned}
\{\text { conn, disc }\} & \rightarrow M \\
\{\text { tree }\} & \rightarrow\{\text { conn, for }\} \\
\{\text { conn, for }\} & \rightarrow\{\text { tree }\} \\
\{\text { cbip }\} & \rightarrow\{b i p\}
\end{aligned}
$$

We do not have any objects at the beginning, so the exploration starts with an empty set $H$. In the following the attributes appearing in the premise of an implication will not be listed in the conclusion again.

The first $\mathcal{L}$-pseudo-intent is the empty set. Therefore the dialogue starts with the question:

$Q:$ Is $\emptyset \rightarrow M$ valid?

$A$ : No. $G_{1}$ has the attributes conn, plan, ham.

Now $H$ contains the object $G_{1}$. In this enlarged context the first $\mathcal{L}$-pseudointent is still the empty set, but on this step we have $\emptyset^{\prime \prime}=\{$ conn, plan, $h a m\}$.

$Q$ : Is $\emptyset \rightarrow\{$ conn, plan, ham $\}$ valid?

A: No. $G_{2}$ has the attributes disc, bip, for, plan.

$G_{2}$ is added to the set $H$.

$Q:$ Is $\emptyset \rightarrow\{$ plan $\}$ valid?

A: No. $G_{3}$ has the attributes conn, comp, eul, ham.

$G_{3}$ is added to $H$. In the context with $H=\left\{G_{1}, G_{2}, G_{3}\right\}$ the empty set is an intent. The next $\mathcal{L}$-pseudo-intent is $\{$ ham $\}$.

$Q:$ Is $\{h a m\} \rightarrow\{\operatorname{con} n\}$ valid?

A: Yes.

The implication $\{h a m\} \rightarrow\{c o n n\}$ is added to $\mathcal{B}_{\mathcal{L}}$ which was empty up to now.

$Q:$ Is $\{e u l\} \rightarrow\{$ conn, comp, ham $\}$ valid?

A: No. $G_{4}$ has the attributes conn, bip, cbip, plan, eul.

$\ldots$ 
During the exploration the graphs $G_{1}$ to $G_{20}$ are given as counterexamples and the following implications are accepted:

$$
\begin{aligned}
\{\text { ham }\} & \rightarrow\{\text { conn }\} \\
\{\text { eul }\} & \rightarrow\{\text { conn }\} \\
\{\text { for }\} & \rightarrow\{\text { bip, plan }\} \\
\{\text { comp }\} & \rightarrow\{\text { conn }\} \\
\{\text { disc, bip, cbip }\} & \rightarrow\{\text { for, plan }\} \\
\{\text { conn, comp, eul }\} & \rightarrow\{\text { ham }\} \\
\{\text { conn, bip, tree, for, plan, ham }\} & \rightarrow\{\text { comp, cbip, eul }\} \\
\{\text { conn, bip, tree, for, plan, eul }\} & \rightarrow\{\text { comp, cbip, ham }\} \\
\{\text { conn, bip, cbip, plan, ham }\} & \rightarrow\{\text { eul }\} \\
\{\text { conn, bip, comp }\} & \rightarrow\{\text { cbip, tree, for, plan }\}
\end{aligned}
$$

These ten implications constitute the $\mathcal{L}$-Duquenne-Guigues-basis $\mathcal{B}_{\mathcal{L}}$. Every implication that is valid in the context can be deduced from them and the four background implications. The Duquenne-Guigues-basis consists of all implications in the $\mathcal{L}$-Duquenne-Guigues-basis and additionally of the first and the fourth background implication and the two implications $\{$ tree $\} \rightarrow$ $\{$ conn, bip, for, plan $\}$ and $\{$ conn, bip, for, plan $\} \rightarrow\{$ tree $\}$. In this example the cardinality of the $\mathcal{L}$-Duquenne-Guigues-basis is just the difference of the cardinalities of the Duquenne-Guigues-basis, but in general it may be larger.

During the exploration there are some implications that can be denied by only one counterexample (up to isomorphism). If we want to determine the general structure of graph theory without bothering with pathological cases, we may confirm some implications that are true for almost all graphs and keep in mind the exceptions. For example we can regard all graphs as exceptions that contradict an implication and are (up to isomorphism) unique in having exactly their attributes.

The beginning of the exploration dialogue remains unchanged. The first difference appears with the question: "Is $\{c o m p\} \rightarrow\{c o n n, e u l$, ham $\}$ valid?", because $G_{6}$ is (up to isomorphism) the only connected complete planar $\mathrm{Ha}$ miltonian graph which is not Eulerian and is therefore not allowed as counterexample. However the implication has to be denied: Graph $G_{6}^{\prime}$ in Fig. 4 serves as new counterexample. The next suggestion $\{$ comp $\} \rightarrow\{$ conn, ham $\}$ will be accepted with the exception $G_{7}$, which is the only complete graph that is not Hamiltonian. In this way the exploration continues. Instead of $G_{14}$ the graph $G_{14}^{\prime}$ will be used as a counterexample for the implication $\{c o n n, e u l, h a m\} \rightarrow\{c o m p\}$, because $G_{14}$ is an exception in the sense defined above.

This approach yields the following list of implications that is a $\mathcal{L}$-basis for all graphs except for $G_{6}, G_{7}, G_{13}, G_{14}$, and $G_{18}$. Behind every implication are listed its exceptions. 

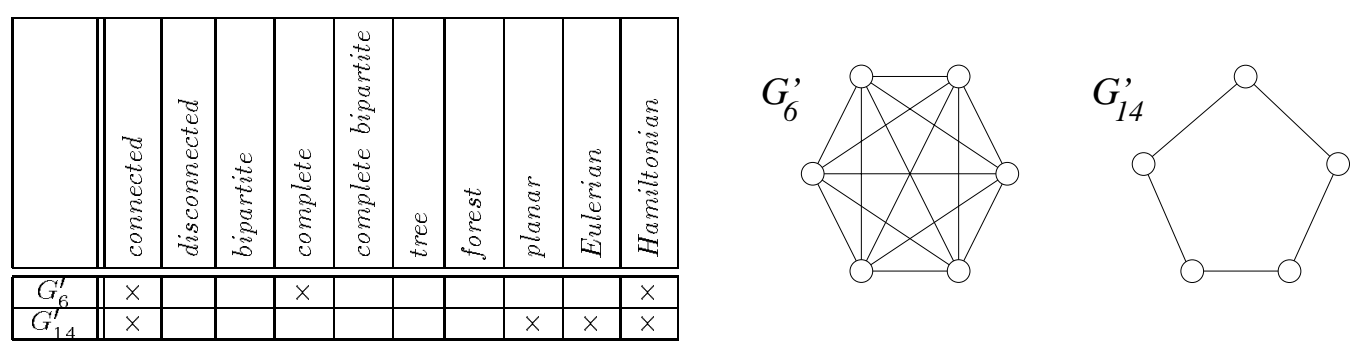

Figure 4: Additional graphs for the exploration with exceptions

$$
\begin{aligned}
& \{h a m\} \rightarrow\{c o n n\} \\
& \{e u l\} \rightarrow\{\text { conn }\} \\
& \{\text { for }\} \rightarrow\{\text { bip, plan }\} \\
& \{\text { comp }\} \rightarrow\{\text { conn, eul, ham }\} \quad\left(G_{6}, G_{7}\right) \\
& \{\text { disc, bip, cbip }\} \rightarrow\{\text { for, plan }\} \\
& \{\text { conn, comp, plan, eul, ham }\} \rightarrow M \\
& \{\text { conn, bip, tree, for, plan, ham }\} \rightarrow M \\
& \{\text { conn, bip, tree, for, plan, eul }\} \rightarrow M \\
& \{\text { conn, bip, cbip, plan, ham }\} \rightarrow\{e u l\} \\
& \{\text { conn, bip, comp, eul, ham }\} \rightarrow M \\
& \left(G_{14}, G_{18}\right) \\
& \left(G_{18}\right) \\
& \left(G_{18}\right) \\
& \left(G_{13}, G_{18}\right) \\
& \left(G_{18}\right)
\end{aligned}
$$

The 6th implication indicates that there exist (up to isomorphism) only two complete planar Hamiltonian graphs: $G_{14}$ and $G_{18}$. That $G_{18}$ is the only Hamiltonian tree and the only Eulerian tree is expressed by 7 th resp. 8th implication. It is also the only Hamiltonian bipartite complete graph (10th implication).

The resulting concept lattice is shown in Fig. 5. The implications valid in this lattice are exactly those which are valid for all graphs except for $G_{6}$, $G_{7}, G_{13}, G_{14}$, and $G_{18}$. The concept lattice of these exceptions is shown in Fig. 6. In particular one can see in the diagram that all exceptions are connected planar graphs.

Attribute exploration determines the $\Lambda$-semilattice that is generated by the attribute concepts. In premise and conclusion only conjunctions of attributes are allowed. Disjunctions become involved in distributive concept exploration (cf. Stumme (1995)), where the complete distributive lattice that is generated by some concepts is interactively determined. 


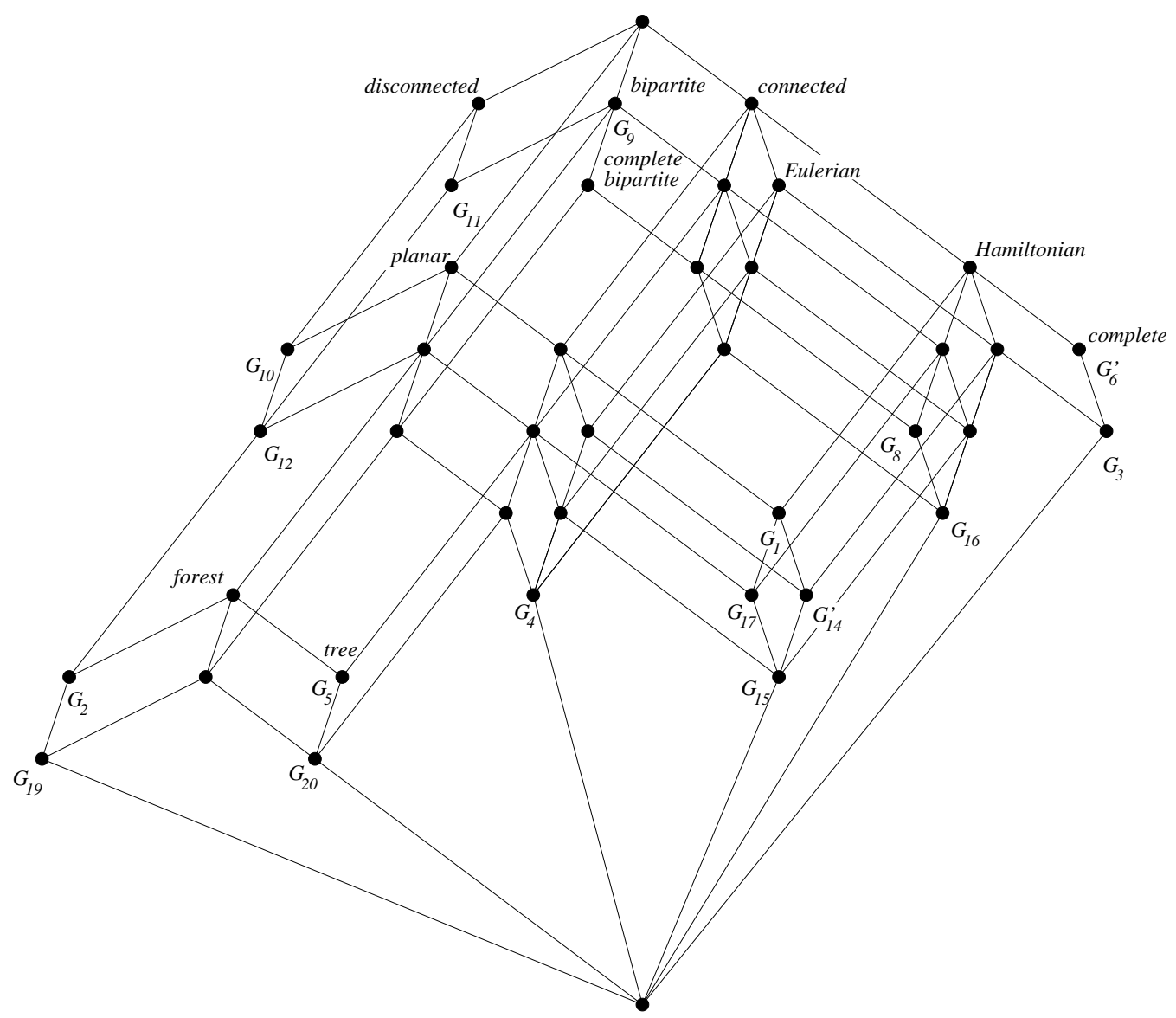

Figure 5: Concept lattice resulting of the exploration with exceptions

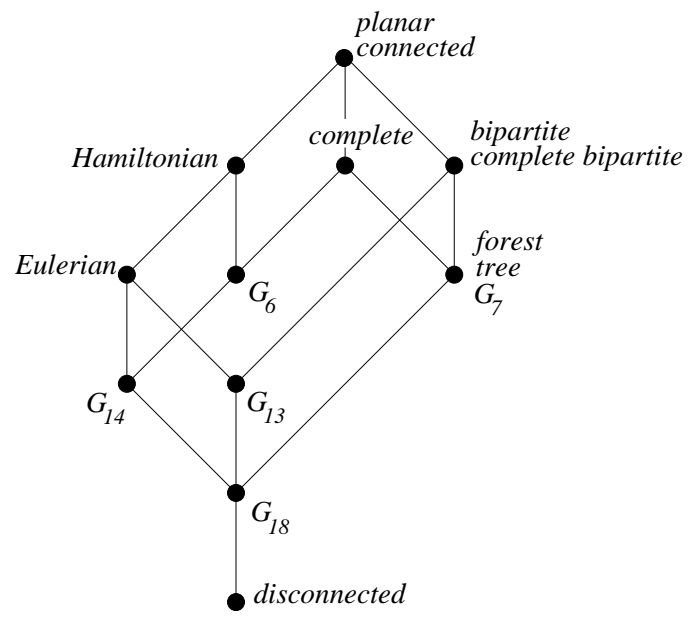

Figure 6: Concept lattice of the exceptions 


\section{References:}

Burmeister, P. (1987): Programm zur formalen Begriffsanalyse einwertiger Kontexte. Technische Hochschule Darmstadt (Latest version 1995 for Atari ST and MS DOS)

Duquenne, V. and Guigues, J.-L. (1986): Familles minimales d' implications informatives resultant d'un tableau de données binaires. Math. Sci. Humaines 95, 5-18

Ehrenberger, P., Heiss, R., Ihringer, Cl., and Vogel, N. (1992): Begriffsverband ausgewählter Graphen. Mittelseminar, TH Darmstadt

Ganter, B. (1987): Algorithmen zur Begriffsanalyse. In: B. Ganter, R. Wille, K. E. Wolff (Eds.): Beiträge zur Begriffsanalyse. B. I.-Wissenschaftsverlag, Mannheim, Wien, Zürich, 241-254

Ganter, B. and Wille, R. (1995): Formale Begriffsanalyse: Mathematische Grundlagen. Springer, Berlin, Heidelberg (To appear)

Stumme, G. (1995): Distributive Concept Exploration - a tool for knowledge acquisition in formal concept analysis. (In preparation)

Wille, R. (1982): Restructuring lattice theory: An approach based on hierarchies of concepts. In: I. Rival (Ed.): Ordered sets. Reidel, Dordrecht-Boston, $445-470$

Wille, R. (1989): Knowledge acquisition by methods of formal concept analysis. In: E. Diday (Ed.): Data analysis, learning symbolic and numeric knowledge. Nova Science Publisher, New York, Budapest, 365-380

WiLson, R. J. (1975): Introduction to graph theory. Longman, London 